\title{
HIGH-FLOW NASAL CANNULAE AND NASAL CPAP USE IN NON-TERTIARY SPECIAL CARE NURSERIES IN AUSTRALIA AND NEW ZEALAND
}

\author{
B. Manley ${ }^{1,2,3}$, L. Owen ${ }^{1,3,4}$, L. Doyle ${ }^{2,5,6}$, P. Davis ${ }^{1,2,3}$
}

${ }^{1}$ Department of Newborn Research, The Royal Women's Hospital, ${ }^{2}$ Department of Obstetrics and Gynaecology, The University of Melbourne, ${ }^{3}$ Murdoch Childrens Research Institute, Parkville, VIC, Australia, ${ }^{4}$ Faculty of Medicine and Dentistry, The University of Bristol, Bristol, UK, ${ }^{5}$ Research Office, The

Royal Women's Hospital, ${ }^{6}$ Department of Paediatrics, The University of Melbourne, Parkville, VIC, Australia

Background: Non-tertiary centres (NTCs) in Australia and New Zealand are increasingly providing noninvasive respiratory support, including high flow nasal cannulae (HFNC) and nasal continuous positive airway pressure (nCPAP), to newborn infants. We aimed to determine the proportion of NTCs in these countries treating newborn infants with HFNC and nCPAP, and how these are used.

Methods: We surveyed public and private NTCs in Australia, and public NTCs in New Zealand, between April and August 2010. The survey, directed at senior medical and nursing staff, consisted of questions regarding unit demographics, HFNC and nCPAP use.

Results: 149 units were contacted. 117 responses were received regarding HFNC use, from $88 \%$ of public hospitals and $64 \%$ of private hospitals surveyed. Ten (7\%) NTCs (nine public, one private) used HFNC in the care of newborn infants. HFNC was used for respiratory distress syndrome from birth (9/10 units), as a weaning mode from nCPAP (5/10), and as treatment for apnoea (3/10). Flow rates used range from 1-8 Litres/minute $(\mathrm{L} / \mathrm{min})$, with typical minimum flow rate $1 \mathrm{~L} / \mathrm{min}$ and maximum $4-6 \mathrm{~L} / \mathrm{min}$. The main perceived advantages of HFNC over nCPAP were ease of nursing care and infant comfort. Many respondents had concerns about the lack of evidence for HFNC use in newborn infants. The rate of nCPAP use has increased to $44 \%$ compared with $17 \%$ in an Australian survey six years earlier.

Conclusions: HFNC is being used in NTCs in Australia and New Zealand, and the use of nCPAP has increased over time. 